\title{
Snapshots of vibrating molecules
}

A spectroscopic imaging method has reached a resolution 1,000 times better than the limits of standard optical imaging techniques - and reveals vibrational modes of molecules previously seen only in computational models. SEE LETTER P.78

\section{ERIC C. LE RU}

$\mathrm{T}$ The resolution of standard optical microscopy is limited to a few hundreds of nanometres, which is much too low to observe ångström-scale atomic motion. Advances in optical methods, combined with electron microscopy, have steadily pushed resolutions down to $15 \mathrm{~nm}$ (ref. 1) and even to below $1 \mathrm{~nm}$ (ref. 2), which is sufficient to resolve the internal structure of a molecule. On page 78, Lee et al. ${ }^{3}$ report further technical developments that enable ångström-scale resolution to be achieved, which they use to image the motion of a vibrating molecule.

Molecules vibrate as if all the bonds between the atoms are little springs. The vibrational motion of a given molecule can be expressed as a linear superposition of many vibrational patterns in which all the atoms oscillate at the same frequency. These patterns are called normal modes. Vibrational motion in a normal mode might simply involve the stretching of one chemical bond, or can involve all the atoms in a molecule. Quantum mechanics tells us that normal modes are quantized - they have well-defined energy values.

Typical vibrational energies of molecules range between 25 millielectronvolts and 0.5 electronvolts. These can be probed either by studying how molecules absorb light (using infrared spectroscopy) or by observing the 'inelastic' scattering of light by molecules (Raman spectroscopy). In the latter case, a photon - typically, from a visible-light laser excites a vibration in the molecule, so that the resulting scattered photon has a lower energy than the incident photon. The energy lost in this process is called the Raman shift, and corresponds exactly to the energy of a vibrational mode. Raman spectra plot the intensity of scattered light (the Raman intensity) against Raman shifts, and thus reveal many of the vibrational energies of a molecule. These spectra can be used as fingerprints to identify molecules. But until now, Raman spectroscopy could reveal only the energies and (if polarized light is used) the general symmetry properties of vibrational motion.

Raman spectroscopy can be combined with microscopy to map out how Raman scattering varies with position, for example on a surface, with submicrometre spatial resolution.
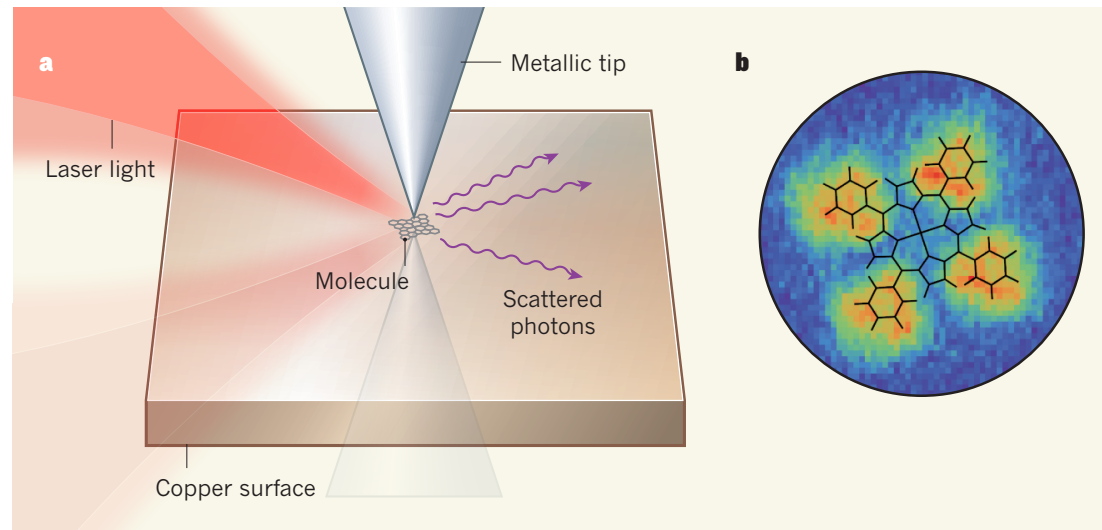

Figure 1 | A spectromicroscopy method for imaging molecular vibrations. a, Lee et al. ${ }^{3}$ studied single molecules anchored on a copper surface. An atomically sharp metallic tip is positioned above the molecule with ångström-scale accuracy, as in a scanning tunnelling microscope. The electromagnetic field of laser light focused on the tip is amplified in the immediate vicinity of the tip's apex, greatly enhancing Raman scattering - a phenomenon in which a photon scattered by a molecule has a lower energy than the incident photon - at this position. b, The resulting images show how the intensity of the scattered light varies with position for a particular vibrational mode of the molecule (red, high-intensity; blue, low intensity). The intensities correlate with the amplitude of atomic vibrations in different regions of the molecule.

However, Raman scattering is typically a weak effect that could not at first be used to detect trace amounts of molecules. This changed 40 years ago with the discovery of surfaceenhanced Raman spectroscopy ${ }^{4}$ (SERS).

SERS exploits the strong interaction between metallic nanostructures, typically made of gold or silver, and visible light. This interaction greatly amplifies the light's electromagnetic field close to the surface of the nanostructures, especially in nanometre-scale gaps between structures, or on the tips of elongated particles. Raman signals from molecules positioned at these 'hotspot' locations are therefore boosted to intensities of between one million and more than one billion times their normal value. This allows the Raman spectrum of single molecules to be measured $d^{5,6}$, providing arguably the ultimate tool for analytical chemistry.

However, because signals from molecules are enhanced only at hotspots, the average gain for an ensemble of molecules is typically smaller than what can be achieved for a single molecule at a hotspot. The challenge of positioning target molecules exactly at hotspots is a subject of much ongoing research.

An alternative approach is to bring the hotspot to the molecule using the metal-coated tip of an atomic force microscope ${ }^{7}$ (AFM) or a scanning tunnelling microscope ${ }^{8}$ (STM), a technique known as tip-enhanced Raman spectroscopy (TERS; Fig. 1). In AFMs and STMs, the tip is passed across a target sample, and images are produced either by measuring the forces between the tip and the sample (AFM), or by measuring the current between the tip and sample when a voltage is applied across them (STM). In STM, the tip can be positioned with ångström-scale accuracy above a molecule of interest lying on a flat substrate.

In TERS, the metal tip is illuminated by a laser to create a highly confined hotspot at its apex, from which the SERS spectrum of a molecule can be measured. Such experiments have reached subnanometre resolution ${ }^{2}$, producing maps of Raman intensity that resolved the internal structure of the studied molecule. They also provided tantalizing hints that slightly different Raman maps were produced for different vibrational modes.

Lee et al. now report TERS images at unprecedented resolutions, down to the angström scale. The key to this advance is to firmly anchor the target molecule to a substrate - in this case, the authors fixed an organic molecule from the porphyrin family to 
a copper surface. They observed that the Raman spectra obtained are different when the tip of the microscope is placed above different regions of the molecule. By plotting the map of Raman intensities for a given vibrational energy, the authors revealed the locations of the atoms most involved in the motion of the associated normal mode. In other words, they obtained snapshots of each vibrational mode (Fig. 1).

These experiments raise questions regarding the mechanisms that enable such a high resolution to be achieved ${ }^{9}$. Hotspots are normally no smaller than nanoscale sizes, which makes it difficult to explain how resolution at much smaller scales can be obtained. Moreover, the electromagnetic theory of continuous media, which explains surface-enhanced Raman scattering, breaks down at the subnanometre scales at which non-local and quantum effects come into play. Full quantum-chemical models of molecules sandwiched between a metal surface and a microscope tip might help us understand how ångström-scale resolution is achieved.
There are limitations to the general use of Lee and colleagues' approach. The experiments are carried out in an ultrahigh vacuum and at a very low temperature (6 kelvin), but such conditions are not accessible to all scientists. Other issues are that only certain pairs of molecules and substrates will provide sufficient anchoring to reach ångström-scale resolution, and the vibrational modes of the molecules might be affected by the substrate. Moreover, the technique is mainly sensitive to motion that is perpendicular to the substrate, rather than to parallel motion.

Nevertheless, overcoming these limitations could open up exciting opportunities, for example in the imaging of biomolecules. One can also envisage combining this ultrahigh-resolution method with approaches that enable ultrafast TERS measurements ${ }^{10}$, to record movies of vibrating molecules. But for now, just being able to experimentally image a vibrational mode will no doubt delight many spectroscopists, who have previously had to rely on theoretical predictions and visualizations.
Eric C. Le Ru is at the MacDiarmid Institute for Advanced Materials and Nanotechnology, School of Chemical and Physical Sciences, Victoria University of Wellington, Wellington 6140, New Zealand. e-mail:eric.leru@vuw.ac.nz

1. Steidtner, J. \& Pettinger, B. Phys. Rev. Lett. 100, 236101 (2008)

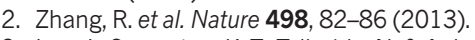

3. Lee, J., Crampton, K. T., Tallarida, N. \& Apkarian, V. A. Nature 568, 78-82 (2019).

4. Le Ru, E. C. \& Etchegoin, P. G. Surface-Enhanced Raman Spectroscopy and Related Plasmonic Effects (Elsevier, 2009).

5. Sonntag, M. D. et al. J. Phys. Chem. C 116, 478-483 (2012).

6. Le Ru, E. C. \& Etchegoin, P. G. Annu. Rev. Phys. Chem. 63, 65-87 (2012).

7. Stöckle, R. M., Suh, Y. D., Deckert, V. \& Zenobi, R. Chem. Phys. Lett. 318, 131-136 (2000).

8. Pettinger, B., Ren, B., Picardi, G., Schuster, R. \& Ertl, G. Phys. Rev. Lett. 92, 096101 (2004).

9. Duan, S. et al. J. Am. Chem. Soc. 137, 9515-9518 (2015).

10.Pozzi, E. A. et al. J. Phys. Chem. Lett. 5, 2657-2661 (2014).

\section{New interactions seen in an ultracold gas}

Another A atom would be attracted to this absence of $B$ atoms, and the end result would again be an attractive mediated interaction between the $\mathrm{A}$ atoms. This simple argument explains why mediated interactions are inherently attractive at short distances. At longer distances, the story is more complicated, because intricate quantum effects can make a mediated interaction repulsive.

DeSalvo and colleagues used a mixture of bosonic caesium-133 atoms and fermionic lithium-6 atoms - bosons have integer spin angular momentum, whereas fermions have half-integer spin. The atoms were confined near the minimum of a harmonic trap, which is a type of potential-energy surface (often simply called a potential) that has a parabolic form. The authors cooled the mixture to extremely
$\mathrm{M}$ any of us have sat in a boat that is being rocked by the waves from another passing vessel. This situation is an example of a mediated interaction: even though the two boats do not touch, they interact through the exchange of waves in the water between them. Mediated interactions have a central role in modern physics. For instance, the fundamental interactions between elementary particles are mediated by particles such as photons ${ }^{1}$. And the attraction between electrons that leads to superconductivity in metals arises from the exchange of vibrations in the surrounding crystal lattice ${ }^{2}$. On page 61 , DeSalvo et al. ${ }^{3}$ report the first observation of mediated interactions in an ultracold mixture of lithium and caesium atoms (Fig. 1a).

Consider a mixture of two kinds of atom, $\mathrm{A}$ and $\mathrm{B}$, and suppose, for clarity, that there are many more $B$ atoms than there are $A$ atoms. Let us first assume that the $\mathrm{A}$ and $\mathrm{B}$ atoms attract each other. Because of this attraction, there would be a high density of B atoms around a given $\mathrm{A}$ atom (Fig. 1b). Another $\mathrm{A}$ atom would be attracted to these $\mathrm{B}$ atoms, and the net effect would be an attractive interaction between the
A atoms that is mediated by the B atoms.

Had we instead assumed that the two kinds of atom repel each other, there would be a low density of B atoms around a given A atom.

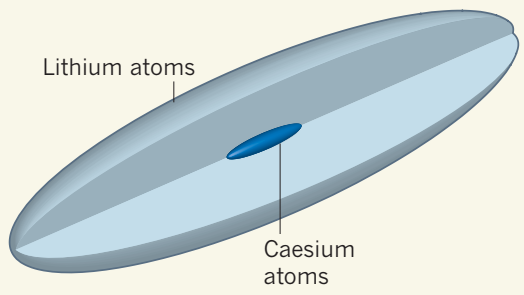

b

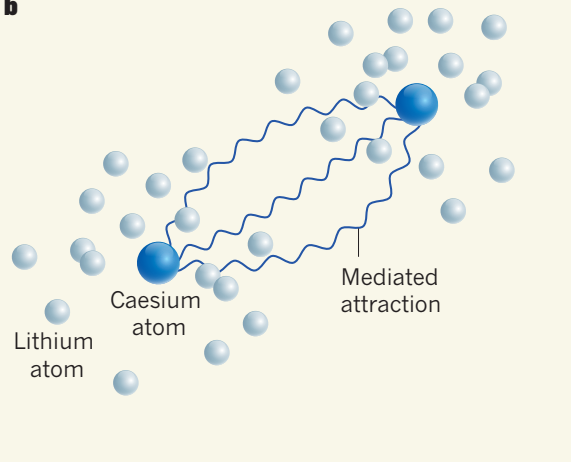

Figure 1 | An ultracold mixture of lithium and caesium atoms. a, DeSalvo et al. ${ }^{3}$ report an experiment in which caesium atoms in a state of matter known a Bose-Einstein condensate are surrounded by lithium atoms in the form of a quantum gas called a Fermi gas. $\mathbf{b}$, When there is an attractive interaction between the two types of atom, there is a high density of lithium atoms around a given caesium atom. Another caesium atom is attracted to this concentration of lithium atoms, resulting in an attractive interaction between the caesium atoms that is mediated by the lithium atoms. A similar argument shows that a repulsive interaction between the two kinds of atom also leads to an attractive mediated interaction between the caesium atoms (not shown). 